\title{
Phenotypic switch in lung interstitial macrophage polarization in an ovalbumin-induced mouse model of asthma
}

\author{
HANXIANG NIE ${ }^{1}$, AILING WANG ${ }^{2}$, QING HE ${ }^{1}$, QIAOYU YANG ${ }^{1}$, LINLIN LIU ${ }^{1}$,

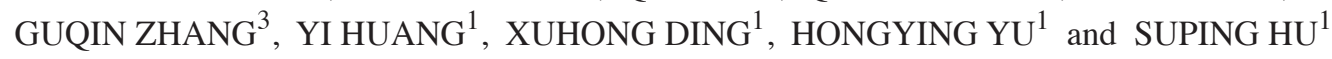 \\ ${ }^{1}$ Department of Respiratory Medicine, Renmin Hospital of Wuhan University; ${ }^{2}$ Nursing Department, \\ Wuhan University School of Health Sciences, ${ }^{3}$ Department of Respiratory Medicine, \\ Zhongnan Hospital of Wuhan University, Wuhan, Hubei 430071, P.R. China
}

Received September 25, 2015; Accepted January 20, 2017

DOI: 10.3892/etm.2017.4699

\begin{abstract}
Macrophage phenotype and function varies according to their polarized state, which in turn is dependent on microenvironmental stimuli. Under normal physiological conditions, lung interstitial macrophages that express interleukin (IL)-10 are considered to serve regulatory roles in the prevention of allergic reactions in the airways. However, the phenotypic profile of lung interstitial macrophages during the pathophysiology of asthma remains unknown. In the current study, the phenotypic characteristics of lung interstitial macrophages were investigated in an ovalbumin (OVA)-induced mouse model of asthma. The patterns of surface markers chemokine ligand and interleukin, and the metabolic enzyme activity of lung interstitial macrophages were investigated using flow cytometry analysis, reverse transcription-quantitative polymerase chain reaction, western blot analysis, and ELISA. It was observed that lung interstitial macrophages derived from OVA-induced asthmatic mice expressed phenotypic markers associated with alternatively activated macrophages (M2), including cluster of differentiation-206, transglutaminase 2, arginase (Arg) 1 and chemokine ligand (CCL)17/CCL22/CCL24 secretion. The M2 macrophages also exhibited increased levels of Arg1 activity and reduced levels of IL-10 expression, relative to macrophages derived from control mice. However, when evaluating the expression
\end{abstract}

Correspondence to: Dr Ailing Wang, Nursing Department, Wuhan University School of Health Sciences, 115 Donghu Road, Wuhan, Hubei 430071, P.R. China

E-mail:nhxbj@sohu.com

Abbreviations: IM, interstitial macrophage; AM, alveolar macrophage; OVA, ovalbumin; Th2, T helper type 2; Arg1, arginase 1; TG2, transglutaminase 2; NO, nitric oxide; iNOS, inducible nitric oxide synthase; IL, interleukin; TNF, tumor necrosis factor; CCL, chemokine ligand; IFN, interferon; CD, cluster of differentiation; BALF, bronchoalveolar lavage fluid

Key words: asthma, lung interstitial macrophage, alternative activation, phenotypic switch of markers associated with classically activated (M1) macrophages, namely inducible nitric oxide synthase and IL-12, it was observed that levels of M1 markers in the interstitial macrophages from asthmatic mice did not differ significantly to those in controls. Collectively, these data suggest that lung interstitial macrophages undergo a phenotypic switch from a regulatory macrophage phenotype under normal conditions to an alternative activation state in OVA-induced asthmatic mice.

\section{Introduction}

Asthma is characterized by chronic airway inflammation, airway hyper-responsiveness and reversible airflow restriction. It is generally considered to be a T helper type 2 (Th2) driven inflammatory disorder (1). However, in addition to the adaptive immune system, the innate immune system is now considered to serve a key role in the pathogenesis of asthma (2).

Macrophages are innate immune cells with key roles in immune defense mechanisms, including tissue homeostasis, primary responses to pathogens, immune resolution, coordination of adaptive immune responses, inflammation and tissue repair (3). Depending on the microenvironment, macrophages can polarize into distinct subsets and the heterogeneity of circulating macrophage precursors (monocytes) may predefine the polarization fate that they assume upon entering a tissue $(4,5)$. Polarized macrophages are broadly classified into two main groups: Classically activated macrophages (M1) and alternatively activated macrophages (M2) $(6,7)$. Induced by interferon (IFN)- $\gamma$ and tumor necrosis factor (TNF)- $\alpha$, M1 macrophages are potent cells that typically attack microorganisms and tumor cells, and are primarily associated with pathological type 1 inflammation, express inducible nitric oxide synthase (iNOS) and the majority of Toll-like receptors (TLRs), and also secrete interleukin (IL)-12, TNF- $\alpha$, IL-1 $\beta$, IL-6, chemokine ligand (CCL) -5 and IFN- $\gamma$-induced protein $10(8,9)$. By contrast, M2 macrophages are induced by IL-4 and IL-13, and serve roles in Th1 inflammatory responses and adaptive immunity, enhancement of Th2 inflammatory responses, angiogenesis and tissue repair (6-9). M2 macrophages are characterized by high expression of the mannose receptor [also known as cluster of differentiation (CD)-206], major histocompatibility class II and a number of 
other signature genes, including arginase 1 (Arg1), transglutaminase 2 (TG2), chitinase-like molecules (Ym1/2 and acid mammalian chitinase) and resistin-like molecule- $\alpha(3,10-12)$. However, in the presence of immune complexes, macrophages polarize into distinct regulatory macrophages $(6,8)$. A key feature of regulatory macrophages is high-level production of the anti-inflammatory cytokine IL-10 (13), and regulatory macrophages have been demonstrated to reduce inflammation and pathological changes in a mouse model of lethal endotoxemia (14) and a mouse model of experimental autoimmune encephalomyelitis (EAE) (15).

In the respiratory tract, macrophages are among the most abundant cells and are grouped into two main populations depending on their localization: Alveolar macrophages (AMs), which line the surface of the alveoli, and interstitial macrophages (IMs), which remain in the space between the alveolar and vascular endothelia (16). While AMs are well characterized (17), the phenotype and in vivo functions of IMs are not fully understood. Previous clinical and in vivo studies have indicated that M2 macrophages are associated with asthma severity. Based on analyses of bronchial biopsies, it has been documented that individuals with asthma exhibit higher percentages of macrophages expressing CD206 (CD206 ${ }^{+}$) and TG2 compared with healthy individuals $(18,19)$. In addition, Kim et al (20) demonstrated that individuals with severe asthma had higher counts of IL-13-positive M2 macrophages in the bronchoalveolar lavage fluid (BALF) compared with healthy individuals. Levels of chitinase molecules and the percentage of $\mathrm{CD}^{206^{+}}$macrophages may also correlate with asthma severity $(19,21)$. Furthermore, a recent study in three murine models of house-dust-mite-induced asthma has indicated that the number of M2 macrophages positively correlates with the severity of airway inflammation (22). By contrast, lower levels of IL-10 have been observed in lung macrophages isolated from asthmatic patients compared to those from healthy individuals (23). It has also been observed that macrophages from severe asthmatic patients produced higher levels of IL-6 and IL-8, relative to individuals with moderate asthma. In addition, IL-10 was undetectable in the patients with severe asthma (24).

Under normal pathological conditions, lung IMs may suppress airway hyper-responsiveness to harmless allergens, principally by producing IL-10 and inhibiting the maturation and migration of dendritic cells from the lymph nodes (25). Macrophages that produce IL-10 are considered to be regulatory macrophages $(15,25)$. Therefore, the present study used an ovalbumin (OVA)-induced mouse model of asthma to determine whether lung IMs undergo a phenotypic switch from a regulatory IL-10-producing macrophage state under normal conditions to an M2-polarized state under asthmatic conditions.

\section{Materials and methods}

Mice. A total of 60 wild-type (WT) female BALB/c mice (6-8 weeks old), with a mean weight of $20 \mathrm{~g}$, were obtained from the Animal Biosafety Level 3 Laboratory of the Center for Animal Experiments at Wuhan University (Wuhan, China). Mice were housed in environmentally controlled and specific pathogen-free conditions $\left(22^{\circ} \mathrm{C}, 12: 12 \mathrm{~h}\right.$ light/dark cycle; $40-70 \%$ humidity) and were given free access to tap water and pellet food. All animal care and handling protocols were approved by the Animal Welfare Committee of Wuhan University.

OVA sensitization and airway challenge. WT BALB/c mice were sensitized intraperitoneally on days 0 and 14 of an airway challenge experiment with $20 \mu \mathrm{g}$ chicken OVA (grade V; Sigma-Aldrich; Merck KgaA, Darmstadt, Germany) emulsified in $2 \mathrm{mg}$ aluminum hydroxide (Pierce; Thermo Fisher Scientific, Inc., Waltham, MA, USA) in $200 \mu$ l phosphate-buffered saline (PBS). Intranasal OVA challenges $(100 \mu \mathrm{g}$ in $50 \mu \mathrm{l}$ PBS) were administered on days 25, 26 and 27. Age- and sex-matched control mice (6 mice per group) from the same batch of wild-type (WT) female BALB/c mice were simultaneously treated with PBS alone. Mice were sacrificed $24 \mathrm{~h}$ after the final OVA challenge with an intraperitoneal injection of sodium pentobarbitone $(100 \mu \mathrm{g} / \mathrm{kg}$; Mintal; Mitsubishi Tanabe Pharma, Osaka, Japan) and their lungs were harvested for analysis.

Cell isolation and culture. Isolated lungs were perfused with $20 \mathrm{ml}$ PBS through the right ventricle. Lungs were then cut into pieces and digested with $1 \mathrm{mg} / \mathrm{ml}$ collagenase I (Invitrogen; Thermo Fisher Scientific, Inc., Waltham, MA, USA) and $0.05 \mathrm{mg} / \mathrm{ml}$ DNase (Roche Diagnostics, Basel, Switzerland) in Hanks' Balanced Salt Solution (Beyotime Institute of Biotechnology, Haimen, China) at $37^{\circ} \mathrm{C}$ for $1 \mathrm{~h}$. The lung mononuclear cells (MNCs) were isolated by centrifugation at $800 \mathrm{x} \mathrm{g}$ for $20 \mathrm{~min}$ at room temperature in a Lymphoprep gradient (density $=1.081 \mathrm{mg} / \mathrm{ml}$; BD Biosciences, Franklin Lakes, NJ, USA). Erythrocytes were lysed by the addition of 10\% volume of Red Blood Cell Lysis Buffer (Sigma-Aldrich; Merck KgaA, Darmstadt, Germany) and incubation for $8 \mathrm{~min}$ at $25^{\circ} \mathrm{C}$ followed by two washes with flow cytometry buffer (00-4222-57; eBioscience Inc., San Diego, CA, USA). Previous studies have demonstrated that lung IMs express the macrophage marker F4/80, but do not express the dendritic cell marker CD11c $(25,26)$. Thus, lung IMs were sorted as $\mathrm{F} 4 / 80^{+} \mathrm{CD} 11 \mathrm{c}^{-}$by flow cytometry. Cell purity following sorting was $\geq 99 \%$, determined by flow cytometry analysis and cytospin analysis using a Rapi-diff Stain set (Clinical Sciences Diagnostics Cc, Johannesburg, South Africa) according to the manufacturer's protocol.

Isolated lung IMs from immunized and control mice were cultured in duplicate at a density of $1 \times 10^{6}$ cells $/ \mathrm{ml}$ in RPMI 1640 medium supplemented with $10 \%$ fetal calf serum, $2 \mathrm{mM}$ L-glutamine, $0.1 \mathrm{mM}$ nonessential amino acids, $50 \mu \mathrm{M}$ mercaptoethanol, $50 \mathrm{U} / \mathrm{ml}$ penicillin and $50 \mathrm{mg} / \mathrm{ml}$ streptomycin (all from Invitrogen; Thermo Fisher Scientific, Inc.) in the presence or absence of OVA supplemented with lipopolysaccharide (LPS; Sigma-Aldrich Merck KgaA; OVA ${ }_{\text {LPS }} ; 100 \mu \mathrm{g} / \mathrm{ml}$ LPS-free OVA and $10 \mathrm{ng} / \mathrm{ml}$ LPS). After $16 \mathrm{~h}$ incubation at $37^{\circ} \mathrm{C}$, cell culture supernatants were collected to measure the secretion levels of the following cytokines and chemokines by ELISA: IL-10, IL-12, CCL17, CCL22 and CCL24.

Flow cytometry analysis. A single cell suspension of lung cells obtained from control and OVA-induced asthmatic mice was resuspended in fluorescence-activated cell sorting (FACS) 
buffer (563503; BD Biosciences, San Jose, CA, USA) at a density of $2 \times 10^{6}$ cells $/ \mathrm{ml}$ at room temperature, according to the manufacturer's protocol. Subsequent staining reactions were performed at $4^{\circ} \mathrm{C}$. To reduce nonspecific binding, cells were first blocked with anti-CD16/CD32 antibody (553141; clone 2.4G2; BD Biosciences) in a staining buffer (Hank's buffer containing $2 \%$ fetal calf serum and $0.1 \%$ sodium azide) on ice for $15 \mathrm{~min}$, then washed once with FACS buffer for $5 \mathrm{~min}$ and labeled with a saturating amount of isotype controls of antibodies on ice for $45 \mathrm{~min}$ in the staining buffer. The antibodies (all 1:100) were as follows: Fluorescein isothiocyanate (FITC)-conjugated anti-F4/80 (11-4801-82; eBioscience Inc), phycoerythrin (PE)-cyano 5 (cy5)-conjugated anti-CD11c (15-0114-82; eBioscience, Inc.), PE-conjugated anti-CD206 (141705; BioLegend, Inc., San Diego, CA, USA), PE-conjugated anti-IL-10 (12-7101-41; eBioscience Inc.), or PE-conjugated anti-iNOS (12-5920-82; eBioscience Inc.). After washing once with FACS buffer on ice for $5 \mathrm{~min}$, to characterize the co-expression of iNOS or CD206 with F4/80 ${ }^{+} \mathrm{CD} 11 \mathrm{c}^{-}$, FITC-conjugated anti-F4/80 and PE-cy5-conjugated anti-CD11c labeled cells were fixed in a fixation buffer $(2 \%$ paraformaldehyde in PBS) at room temperature for $10 \mathrm{~min}$, then incubated with PE-conjugated CD206 (141705; 1:100; BioLegend, Inc.), PE-conjugated anti-IL-10 (12-7101-41; 1:100; eBioscience Inc.), or PE-conjugated anti-iNOS (12-5920-82; 1:100; eBioscience Inc.) on ice for $45 \mathrm{~min}$ in the staining buffer following membrane permeabilization with a Leucoperm kit (AbD Serotec, Raleigh, NC, USA), according to the manufacturer's protocol. Cells were sorted and analyzed using an EPICS Altra Flow Cytometer (Epics Altra II; Beckman Coulter, Inc., Brea, CA, USA).

Detection of IL-10, IL-12, CCL17, CCL22 and CCL24 by ELISA. ELISA was used to quantify the secretion levels of the cytokines IL-10 and IL-12 and chemokines CCL17, CCL22 and CCL24 in the culture supernatant of lung IMs. IL-10 (catalogue number: BMS614/2) and IL-12 (catalogue number: BMS616) ELISA kits were purchased from eBioscience, Inc. and CCL17 (catalogue number: MCC170), CCL22 (catalogue number: MCC220) and CCL24 (catalogue number: DY528) ELISA kits were purchased from R\&D Systems, Inc. (Minneapolis, MN, USA). All assays were performed according to the manufacturers' protocols.

Reverse transcription-quantitative polymerase chain reaction $(R T-q P C R)$. Total RNA was extracted from FACS-sorted lung IMs using TRIzol reagent (Applied Biosystems; Thermo Fisher Scientific, Inc.), according to the manufacturer's protocol. Isolated RNA was then purified using an RNeasy Microprep kit (Qiagen $\mathrm{GmbH}$, Hilden, Germany). cDNA was synthesized using a SuperScript II reverse transcriptase (Invitrogen; Thermo Fisher Scientific, Inc.), according to the manufacturer's protocol. qPCR was performed using a LightCycler ${ }^{\circledR}$ FastStart DNA Master ${ }^{\text {PLUS }}$ SYBRGreen I reaction mix (Roche Diagnostics) on a Lightcycler 480II (Roche Diagnostics). The levels of mRNA expression for $i N O S$, Argl, TG, and IL-10 were measured using primers purchased from Thermo Fisher Scientific, Inc. The primer sequences were as follows: For iNOS, forward, 5'-CGGAGCCTTTAGACCTCAACAGA-3' and reverse, 5'-TAGGACAATCCACAACTCGCTCC-3', for Arg1, forward, 5'-TGGGAAGACAGCAGAGGAGGT G-3' and reverse, 5'-GCTTATGGTTACCCTCCCGTTG-3', for IL-10, forward, 5'-TGGACAACATACTGCTAACCG AC-3' and reverse 5'-CCTGGGGCATCACTTCTACC-3', for TG2, forward, 5'-TTCCGGCTGACTCTGTACTTCGAG-3' and reverse, 5'-ACATTGTCCTGTTGGTCCAGCACT-3' and for GAPDH, forward, 5'-AGGAGCGAGACCCCACTA ACA-3' and reverse, 5'-AGGGGGGCTAAGCAGTTGGT-3'. The PCR protocol consisted of $60 \mathrm{sec}$ at $95^{\circ} \mathrm{C}$, followed by 40 cycles of $15 \mathrm{sec}$ at $95^{\circ} \mathrm{C}, 15 \mathrm{sec}$ at $59^{\circ} \mathrm{C}$ and $45 \mathrm{sec}$ at $72^{\circ} \mathrm{C}$. All data were analyzed using SDS 2.2 software (Applied Biosystems; Thermo Fisher Scientific, Inc.). Gene expression was quantified as the average of triplicate samples after standard curve interpolation (relative standard curve method) (27) and normalization to GAPDH gene expression.

Western blot analysis of Arg 1, iNOS, and IL-10 protein expression. Single cell suspensions of lung IMs isolated from control and OVA-induced asthmatic mice were prepared and pooled across mice within each group. Cells were lysed for $20 \mathrm{~min}$ at $4^{\circ} \mathrm{C}$ in radioimmunoprecipitation assay buffer (10 mM phosphate buffer pH 7.4, $150 \mathrm{mM}$ $\mathrm{NaCl}, 1 \% \mathrm{NP}-40,0.5 \%$ sodium deoxycholate, $0.1 \%$ SDS; R-2417; AG Scientific Inc., San Diego, CA, USA), according to the manufacturer's protocol. Lysates were then collected following centrifugation at $12,000 \times \mathrm{g}$ at $4^{\circ} \mathrm{C}$ for $20 \mathrm{~min}$. Protein concentration was assayed using a Bicinchoninic Acid (BCA) Protein Assay kit (Pierce; Thermo Fisher Scientific, Inc.) and whole lysates were mixed with 4X SDS loading buffer (125 mM Tris-hydrogen chloride, 4\% SDS, $20 \%$ glycerol, $100 \mathrm{mM}$ dithiothreitol and $0.2 \%$ bromophenol blue) in a 1:3 ratio. Samples were heated at $100^{\circ} \mathrm{C}$ for $5 \mathrm{~min}$ and separated on SDS-polyacrylamide gels $(10 \times 10 \mathrm{~cm}$ by $0.75 \mathrm{~mm}$ ) containing 4 and $12.5 \%$ acrylamide in the stacking and separating gels, respectively. A total of $50 \mu \mathrm{g}$ protein was loaded per lane. Separated proteins were then transferred to polyvinylidene difluoride membranes in a solution of transfer buffer $(25 \mathrm{mM}$ Tris, $192 \mathrm{mM}$ glycine, $0.01 \%$ SDS, $20 \%$, v/v, methanol) at $200 \mathrm{~mA}$ for $2 \mathrm{~h}$. After transfer, the membranes were rinsed for $5 \mathrm{~min}$ with wash buffer and $0.02 \%$ Tween-20. Wash buffer was removed and the membranes were incubated with $5 \%$ blocking buffer (blotting grade blocker non-fat dry milk; Bio-Rad Laboratories, Inc., Hercules, CA, USA) for $1 \mathrm{~h}$ at room temperature. Membrane blots were probed with primary antibodies overnight at $4^{\circ} \mathrm{C}$, then incubated with a corresponding horseradish peroxidase (HRP)-conjugated secondary antibody for $1 \mathrm{~h}$ at room temperature (RT). Proteins were visualized using an enhanced chemiluminescent system (GE Healthcare Life Sciences, Chalfont, UK) The following primary antibodies were diluted to 1:500 in TBST buffer (10 mM Tris $\mathrm{HCl}, 150 \mathrm{mM} \mathrm{NaCl}, 0.1 \%$ Tween-20) and used: Rabbit anti-mouse anti-Arg1 (610709; BD Biosciences), -iNOS (14-5920-82; eBioscience, Inc.), -IL-10 (14-71-01; eBioscience, Inc.) and -GAPDH (14-9523-82; eBioscience, Inc.). The HRP-conjugated secondary antibody was anti-rabbit (18-4818-82; eBioscience, Inc.). Optical densities (ODs) of bands were measured using Scion Image software (Scion Image Beta 4.02 for Windows; Scion Corporation, 
Frederick, MD, USA). The ODs of Arg1, iNOS and IL-10 were normalized to that of GAPDH.

iNOS and Argl activity assays. FACS-sorted lung IMs isolated from control and OVA-induced asthmatic mice were plated in triplicate into 96 -well plates at a density of $2 \times 10^{5}$ cells/well and $2 \times 10^{4}$ cells/well for iNOS and Arg1 activity assays, respectively. Isolated cells were cultured in RPMI 1640 medium supplemented with $10 \%$ fetal calf serum, $2 \mathrm{mM}$ 1-glutamine, $1 \mathrm{mM}$ sodium pyruvate, $0.1 \mathrm{mM}$ nonessential amino acids, $50 \mu \mathrm{M} \beta$-mercaptoethanol, $50 \mu \mathrm{g} / \mathrm{ml}$ streptomycin, and $50 \mathrm{IU} / \mathrm{ml}$ penicillin (all Invitrogen; Thermo Fisher Scientific, Inc.) at $37^{\circ} \mathrm{C}$ for $48 \mathrm{~h}$. Levels of nitric oxide (NO) in the culture supernatant were measured based on the Griess reaction using Griess reagent (G2930; Promega Corporation, Madison, WI, USA) according to manufacturer's protocol and absorbance was measured at a wavelength of $570 \mathrm{~nm}$ with a microplate reader (MR 710, Dynatech Scientific Labs, El Paso, TX, USA) to evaluate iNOS activity. Arg1 activity was determined by measuring the enzymatic conversion of arginine into urea in the cell culture supernatants using a DARG-200 QuantiChrom ${ }^{\text {TM }}$ Arginase Assay kit (BioAssay Systems, Hayward, CA, USA). Protein concentration was determined using a BCA Protein Assay kit (Pierce; Thermo Fisher scientific, Inc., Hemel Hempstead, UK).

Statistical analysis. All data are expressed as the mean \pm standard deviation of at least three independent experiments. Student's t-test was used to determine significant differences between two groups. Data were analyzed using GraphPad Prism 5 software (GraphPad Software Inc., La Jolla, CA, USA) and $\mathrm{P}<0.05$ was considered to indicate a statistically significant difference.

\section{Results}

$\mathrm{CD}^{206^{+}} \mathrm{F}^{\prime} / 80^{+} \mathrm{CD} 11 \mathrm{c}^{-}$macrophages accumulate in the lungs of OVA-induced asthmatic mice. Previous studies have demonstrated that lung IMs express the macrophage marker F4/80 but do not express the dendritic cell marker CD11c $(25,26)$. To further characterize IMs in an OVA-induced mouse model of asthma, whole lungs from BALB/c OVA-induced asthmatic mice and PBS-treated control mice were digested $24 \mathrm{~h}$ after the final OVA challenge (day 27 post-model establishment; $100 \mu \mathrm{g}$ in $50 \mu \mathrm{l}$ PBS). Isolated cells were subsequently stained for $\mathrm{F} 4 / 80$ and CD11c and analyzed by flow cytometry. It was observed that the percentage of $\mathrm{F} 4 / 80^{+} \mathrm{CD} 11 \mathrm{c}^{-}$macrophages was significantly higher in OVA-induced asthmatic mice relative to that in control mice $(\mathrm{P}<0.01$; Fig. 1A and $\mathrm{B})$. It was also observed that lung IMs were polarized towards an M2 macrophage phenotype. This was demonstrated by a significant increase in the expression of CD206, as a marker of M2 macrophages, on IMs ( $\mathrm{F} 4 / 80^{+} \mathrm{CD} 11 \mathrm{c}^{-}$cells) isolated from OVA-induced asthmatic mice, relative to control mice $(\mathrm{P}<0.01$; Fig. $1 \mathrm{C}$ and $\mathrm{D})$. By contrast, the number of $\mathrm{IL}-10^{+} \mathrm{F} 4 / 80^{+} \mathrm{CD} 11 \mathrm{c}^{-}$macrophages, which are considered to be regulatory macrophages, significantly decreased in OVA-induced asthmatic mice relative to control mice $(\mathrm{P}<0.01$; Fig. $1 \mathrm{E}$ and F). Furthermore, lung IMs from control and OVA-induced asthmatic mice expressed
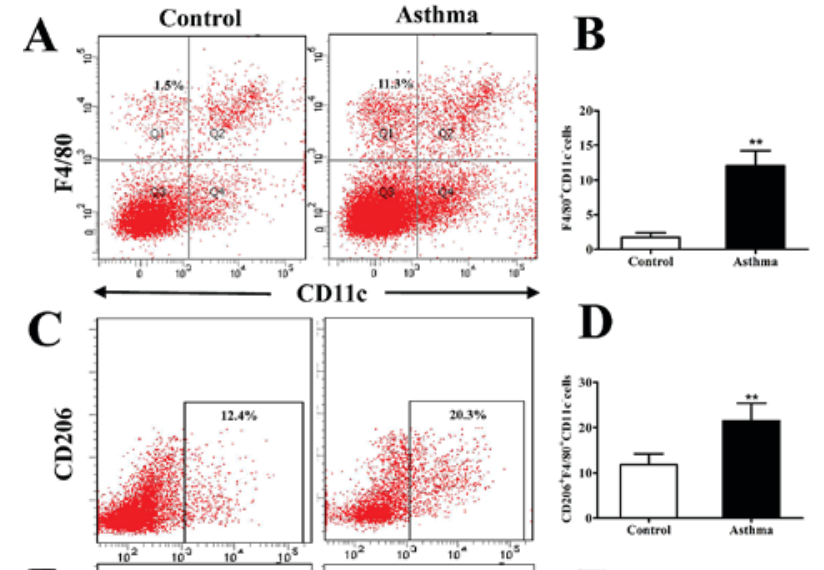

D
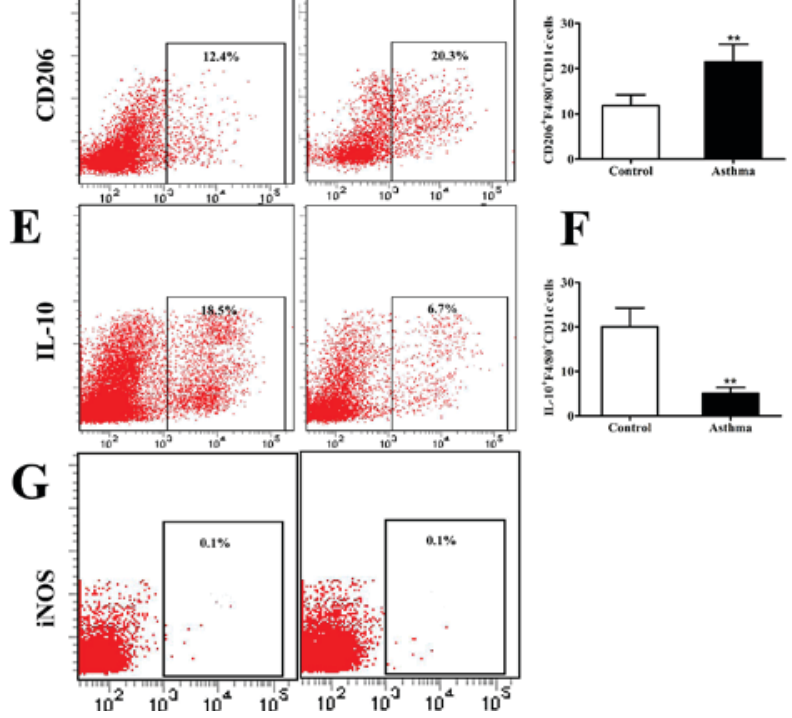

Figure 1. Flow cytometry analysis of $\mathrm{CD}_{2} 06^{+} \mathrm{F} 4 / 80^{+} \mathrm{CD} 11 \mathrm{c}^{-}$cells in the lungs of OVA-induced asthmatic mice. (A) Flow cytometry was used to identify $\mathrm{F} 4 / 80^{+}$and $\mathrm{CD} 11 \mathrm{c}^{-}$lung IMs isolated from the lungs of OVA-induced asthmatic mice and PBS-treated control mice. Boxes indicate the gating region of lung IMs (F4/80 ${ }^{+} \mathrm{CD} 11 \mathrm{c}^{-}$; gate B). (B) Relative percentages of $\mathrm{F} 4 / 80^{+} \mathrm{CD} 11 \mathrm{c}$ cells in control and OVA-induced asthmatic mice. (C) Detection of lung IMs positive for the cell surface marker CD206. Boxes indicate the gating region of $\mathrm{CD}^{2} 06^{+} \mathrm{F} 4 / 80^{+} \mathrm{CD} 11 \mathrm{c}^{-}$cells (gate $\mathrm{C}$ ). (D) Relative percentages of $\mathrm{CD} 206^{+} \mathrm{F} 4 / 80^{+} \mathrm{CD} 11 \mathrm{c}^{-}$cells in control and OVA-induced asthmatic mice. (E) Detection of lung IMs positive for IL-10. Boxes indicate the gating region of $\mathrm{IL}-10^{+} \mathrm{F} 4 / 80^{+} \mathrm{CD} 11 \mathrm{c}^{-}$cells (gate $\mathrm{C}$ ). (F) Percentages of lung IMs expressing IL-10 in control and OVA-asthmatic mice $(\mathrm{G})$ Detection of lung IMs positive for iNOS. Boxes indicate the gating region of iNOS ${ }^{+} \mathrm{F} 4 / 80^{+} \mathrm{CD} 11 \mathrm{c}^{-}$cells (gate P2). Percentages on each dot plot represent positively stained cells. All data are presented as the mean \pm standard deviation, $n=6$. ${ }^{* *} \mathrm{P}<0.01$ vs. control. CD, cluster of differentiation; F4/80, epidermal growth factor family cell surface antigen; IM, interstitial macrophages; IL-10, interleukin-10; OVA, ovalbumin; iNOS, inducible nitric oxide synthase; PBS, phosphate-buffered saline; PE-cy5, phycoerythrin-cyano 5 dye; FITCH, fluorescein isothiocyanate.

markedly low levels of iNOS (Fig. 1G), which is a marker of classically activated (M1) macrophages. Collectively, these results indicate that M2-polarized lung IMs accumulate in the lungs of an OVA-induced asthma mouse model.

Expression of signature genes associated with M2 $\mathrm{F} / 8 \mathrm{O}^{+} \mathrm{CD} 11 \mathrm{c}^{-}$macrophages is increased in the lung IMs of OVA-induced asthmatic mice. Lung IMs isolated from control and OVA-induced asthmatic mice were sorted $24 \mathrm{~h}$ after the final OVA challenge and their RNA was isolated. The expression of mRNA associated with classically and alternatively activated macrophages, along with IL-10 mRNA, were measured by RT-qPCR. It was observed that levels of Arg1 and TG2 mRNA, as markers of alternatively activated macrophages, were significantly increased relative to control mice 

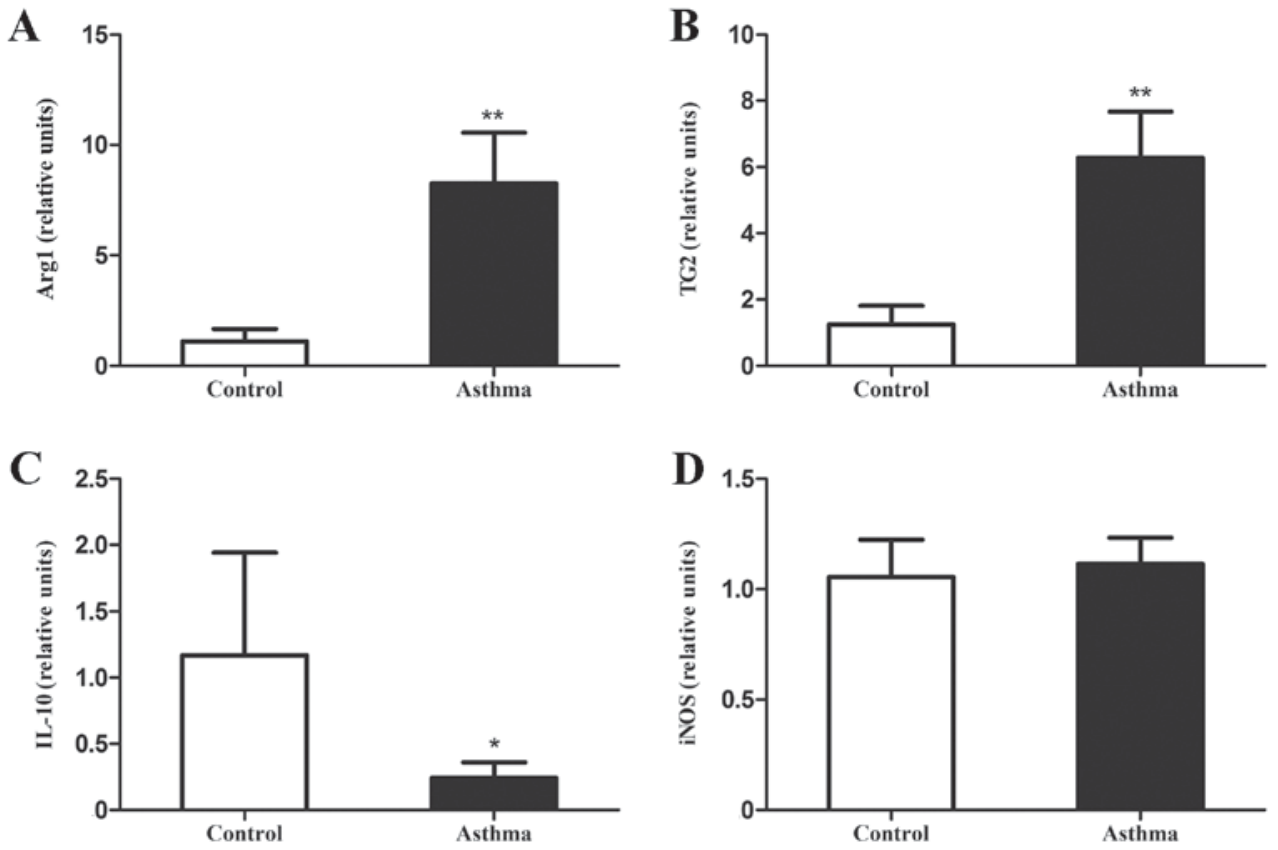

Figure 2. Expression of genes associated with alternatively activated macrophages in the lungs of OVA-induced asthmatic mice. Lung IMs from OVA-induced asthmatic mice and PBS-treated control mice were sorted $24 \mathrm{~h}$ after the final OVA challenge (day 27 post-model establishment; $100 \mu \mathrm{g}$ in $50 \mu 1$ PBS) and the levels of (A) Arg1, (B) TG2, (C) IL-10 mRNA and (D) iNOS mRNA, as markers of classically activated macrophages, were measured by reverse transcription-quantitative polymerase chain reaction. Expression of Arg1, TG2, iNOS, and IL-10 was normalized to GAPDH expression. Data are presented as the mean \pm standard deviation, $\mathrm{n}=6 .{ }^{*} \mathrm{P}<0.05$ and ${ }^{* *} \mathrm{P}<0.01$ vs. control. OVA, ovalbumin; IM, interstitial macrophage; Arg1, arginase 1; TG2, transglutaminase 2 ; iNOS, inducible nitric oxide synthase; IL-10, interleukin-10; PBS, phosphate-buffered saline.

( $\mathrm{P}<0.01 ;$ Fig. 2A and B). In addition, IL-10 mRNA expression, as a primary feature of regulatory macrophages, was significantly decreased in OVA-induced asthmatic mice relative to control mice $(\mathrm{P}<0.05$; Fig. $2 \mathrm{C})$. However, levels of iNOS mRNA, as a marker of classically activated macrophages, did not significantly differ between OVA-induced asthmatic mice and control mice ( $\mathrm{P}>0.05$; Fig. 2D).

Expression of signature proteins associated with M2

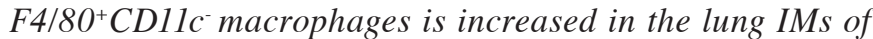
OVA-induced asthmatic mice. It was observed following western blot analysis (Fig. 3A) that the levels of Arg1 protein in the lung IMs of OVA-induced asthmatic mice were significantly increased, relative to control mice ( $\mathrm{P}<0.01$; Fig. 3B). However, levels of iNOS protein did not significantly differ between the two groups ( $\mathrm{P}>0.05$; Fig. $3 \mathrm{C}$ ). In addition, the expression of IL-10 in the lung IMs of OVA-induced asthmatic mice was significantly decreased, relative to control mice $(\mathrm{P}<0.01$; Fig. 3D). Collectively, these data indicate that lung IMs in an OVA-induced asthma mouse model exhibit increased expression of signature genes and proteins associated with M2 polarization.

IL-10 is reduced while chemokine secretion is increased in lung IMs of OVA-induced asthmatic mice. To determine whether lung IMs produce relevant inflammatory factors of the different macrophage phenotypes (cytokines IL-10 and IL-12 and chemokines CCL17, CCL22 and CCL24), lung IMs isolated from control and OVA-induced asthmatic mice were cultured in the presence or absence of OVA $\mathrm{LPS}_{\text {. After }}$ $16 \mathrm{~h}$, culture supernatants were collected and analyzed for cytokine and chemokine expression. It was observed that
IL-10 concentration was significantly lower in the culture supernatant of unstimulated lung IMs (no OVA LPS $_{\text {group) }}$ of OVA-induced asthmatic mice, relative to control mice (no $\mathrm{OVA}_{\mathrm{LPS}}$ group; $\mathrm{P}<0.01$; Fig. $4 \mathrm{~A}$ ). The concentration of IL-12, as a pro-inflammatory cytokine released by classically activated macrophages (28) did not significantly differ in the culture supernatants of unstimulated lung IMs of OVA-induced asthmatic mice, relative to control mice ( $\mathrm{P}>0.05$; Fig. 4B). The concentrations of CCL17, CCL22 and CCL24, as chemokines typically secreted by alternatively activated macrophages (23), were significantly higher in the culture supernatant of unstimulated lung IMs from OVA-induced asthmatic mice, relative to unstimulated lung IMs from control mice ( $\mathrm{P}<0.01$; Fig. $4 \mathrm{C})$. Stimulation with $\mathrm{OVA}_{\mathrm{LPS}}$ had no significant effect on the levels of IL-10 produced by the lung IMs of OVA-induced asthmatic mice, relative to unstimulated cells in the OVA-asthmatic group (P>0.05; Fig. 4A). By contrast, $\mathrm{OVA}_{\mathrm{LPS}}{ }^{-}$stimulated lung IMs from control mice produced significantly more IL-10 than unstimulated control cells $(\mathrm{P}<0.01$; Fig. 4A). In addition, stimulation with $\mathrm{OVA}_{\mathrm{LPS}}$ had no significant effect on the levels of IL-12 produced by lung IMs of OVA-induced asthmatic or control mice, relative to unstimulated lung IMs in their respective groups (both $\mathrm{P}>0.05$; Fig. 4B). Stimulation with $\mathrm{OVA}_{\mathrm{LPS}}$ also had no significant effect on the production of CCL17, CCL22 or CCL24 from lung IMs of control mice (P>0.05; Fig. 4C). However, $\mathrm{OVA}_{\mathrm{LPS}}$-stimulated lung IMs produced significantly more CCL17, CCL22 and CCL24 than unstimulated lung IMs from OVA-induced asthmatic mice (all $\mathrm{P}<0.01$; Fig. 4C). Collectively, these data suggest that the quiescent phenotype of lung IMs switches to an M2 polarized state in an OVA-induced asthma mouse model. 
A

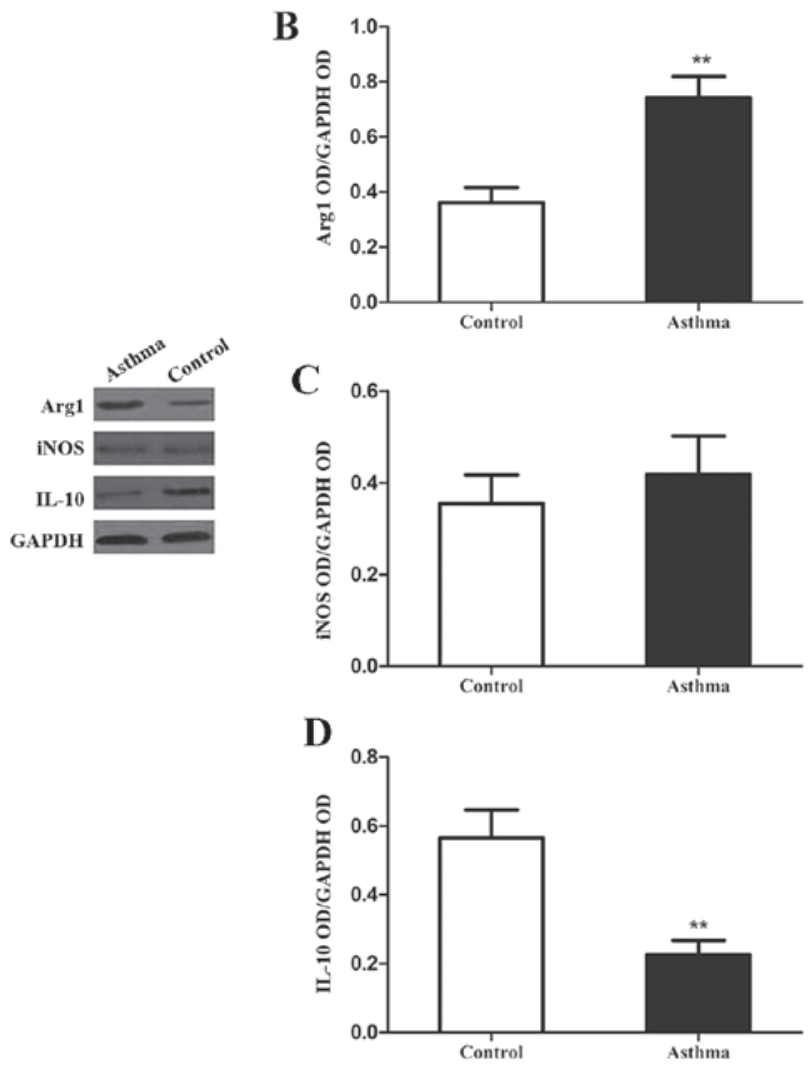

Figure 3. Expression of Arg1, iNOS and IL-10 in lung IMs of OVA-induced asthmatic mice. Lung IMs from OVA-induced asthmatic mice and PBS-treated control mice were sorted $24 \mathrm{~h}$ after the final OVA challenge (day 27 post-model establishment; $100 \mu \mathrm{g}$ in $50 \mu \mathrm{l}$ PBS) and (A) western blot analysis was used to measure Arg1, IL-10 and iNOS protein expression. GAPDH was used as a protein loading control and images are representative of 6 mice in each group. ODs of (B) Arg1, (C) iNOS and (D) IL-10 levels normalized to GAPDH. Data are presented as the mean \pm standard deviation, $\mathrm{n}=6 .{ }^{* *} \mathrm{P}<0.01$ vs. control. Arg1, arginase 1 ; iNOS, inducible nitric oxide synthase; IL-10, interleukin-10; OVA, ovalbumin; IM, interstitial macrophage; PBS, phosphate-buffered saline; OD, optical density.

Argl activity is in the lung IMs of OVA-induced asthmatic mice. Increased arginase activity is also a characteristic of alternatively activated macrophages (29). Therefore, the present study measured the levels of alternatively activated macrophages in the lungs of an OVA-induced asthmatic mouse model through ex vivo assays of Arg1 and iNOS catalytic activity (Fig. 5). It was observed that Arg1 activity in the lung IMs of OVA-induced asthmatic mice was significantly elevated relative to control mice $(\mathrm{P}<0.01)$. In addition, iNOS activity did not significantly differ between the control and asthmatic groups. This suggests that the Arg1/iNOS balance in the lung IMs of an OVA-induced asthma mouse model is skewed towards an M2 polarization state.

\section{Discussion}

The innate immune system is associated with the induction and progression of airway diseases, including asthma. Although macrophages are a key part of the innate immune system, their contribution to the pathogenesis of asthma is currently unknown. A previous study indicated that alternatively activated macrophages and the inflammatory molecules they produce are associated with asthma (30). AMs and IMs are the two main populations of macrophages in the lungs and occupy distinct compartments (16). The characteristics of AMs have been widely studied (17), however the IM phenotype and its in vivo functions are not fully understood. Therefore, the present study evaluated the subtypes and expression profiles of lung IMs in a mouse model of OVA-induced asthma. It was principally demonstrated that the lung IMs of OVA-induced asthmatic mice express hallmark genes of macrophage polarization towards an alternatively activated state (M2), thus implying a causative role of these cells in the pathogenesis of asthma.

Macrophage activation is a dynamic process, in which cells that are initially involved in pro-inflammatory and cytotoxic reactions subsequently participate in the resolution of inflammation and wound healing (7,30-33). Therefore, macrophage differentiation is an adaptable process, enabling macrophages to modulate their response to changing microenvironments $(7,31,33)$. Macrophages are activated by different stimuli and the cells subsequently express distinct patterns of chemokines, cytokines, surface markers and metabolic enzymes that ultimately generate the diversity of macrophage function observed in inflammatory and noninflammatory settings $(6,28)$. Macrophage activation is defined by two distinct polarization states: M1 (classically activated macrophages) and M2 (alternatively activated macrophages) $(6,28)$. Other macrophage subtypes also exist, including regulatory macrophages $(6,8)$. Macrophage activation into an M2 polarization state involving tumor-associated macrophages or parasitic infections has been previously documented in different tumor settings, and is considered to modulate tissue repair and prevent excess inflammation $(30,34)$. In the present study, lung IMs exhibited increased levels of CD206 expression, as a surface marker of alternatively activated macrophages (31), indicating that M2-polarized lung IMs accumulate in the lungs of OVA-induced asthmatic mice.

The balance between arginase and iNOS activity is an important distinction between M1 and M2 polarization states. Both arginase and iNOS utilize L-arginine, though only arginase production is increased in M2-polarized macrophages (35). In addition, arginase blocks iNOS activity through a number of mechanisms, including outcompeting iNOS for the arginine substrate, which is required for NO production (35). Based on direct measurements of arginase activity and gene expression, including for Arg1 and TG2, as markers of M2 macrophages, the present study demonstrated that lung IMs of an OVA-induced asthma mouse model exhibit increased arginase activity, compared with control mice. Furthermore, it was observed that chemokines typically produced by alternatively activated macrophages, namely CCL17, CCL22 and CCL24, are produced in greater quantities by lung IMs from OVA-induced asthmatic mice, relative to controls. However, lung IMs from control and OVA-induced asthmatic mice exhibited similar levels of iNOS, along with similar levels of IL-12 secretion. In addition, levels of iNOS activity in the lung IMs of OVA-induced asthmatic mice were similar to controls. Collectively, these data suggest that lung IMs in an OVA-induced asthma mouse model are polarized towards an alternative activation phenotype. 
A

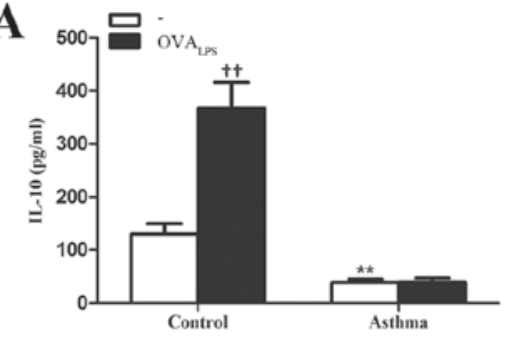

B

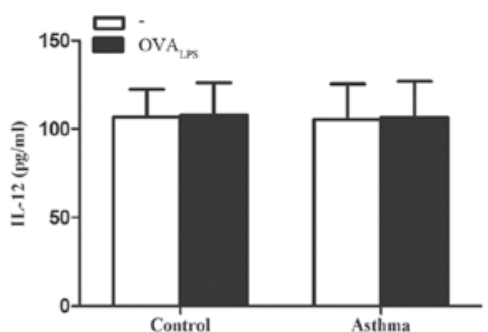

C
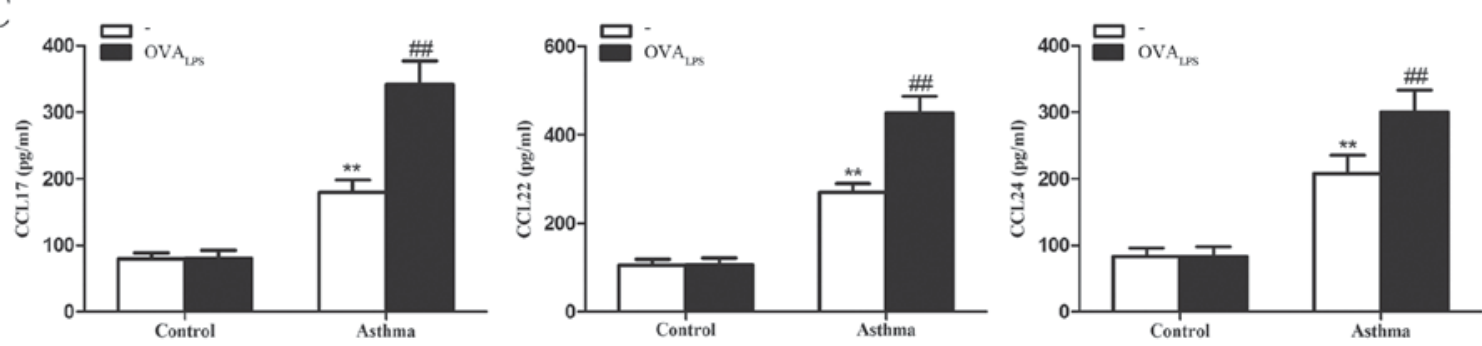

Figure 4. Expression of cytokines IL-10 and IL-12 and chemokines CCL17, CCL22 and CCL24 in lung IMs of OVA-induced asthmatic mice. Lung IMs from OVA-induced asthmatic mice and PBS-treated control mice were sorted $24 \mathrm{~h}$ after the final OVA challenge (day 27 post-model establishment; $100 \mu \mathrm{g}$ in $50 \mu \mathrm{l}$

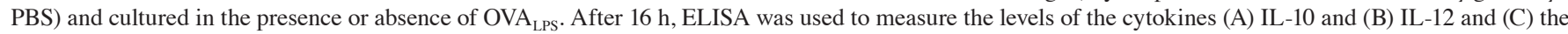
chemokines CCL17, CCL22 and CCL27 in the culture supernatant. Data are presented as the mean \pm standard deviation, $n=6$. ${ }^{* *} \mathrm{P}<0.01 \mathrm{vs}$. non $\mathrm{OVAA} \mathrm{LPs}_{\mathrm{LS}}$-stimulated control mice; ${ }^{\dagger \mathrm{P}}<0.01$ vs. non $\mathrm{OVA}_{\mathrm{LPS}}$-stimulated control mice; ${ }^{\# \#} \mathrm{P}<0.01$ vs. non $\mathrm{OVA}_{\mathrm{LPS}}$-stimulated asthmatic mice. IL, interleukin; CCL, chemokine ligand; OVA, ovalbumin; $\mathrm{OVA}_{\mathrm{LPS}}$, lipopolysaccharide supplemented OVA; IM, interstitial macrophage; PBS, phosphate-buffered saline.
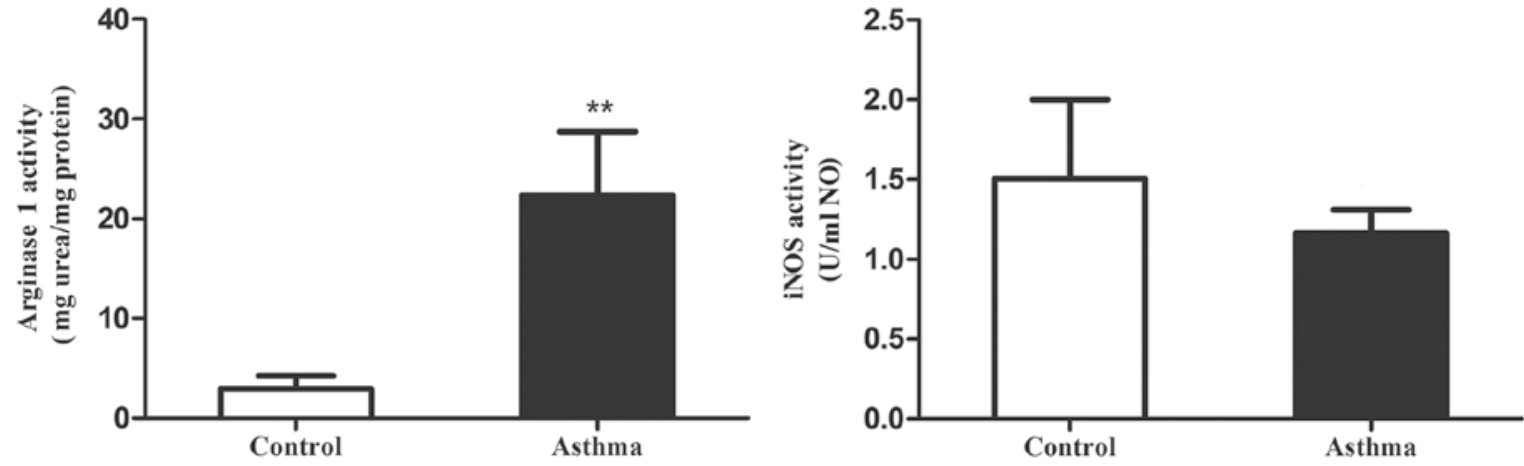

Figure 5. Arg1 and iNOS activity in lung IMs of OVA-induced asthmatic mice. Lung IMs from OVA-induced asthmatic mice and PBS-treated control mice were sorted $24 \mathrm{~h}$ after the final OVA challenge (day 27 post-model establishment; $100 \mu \mathrm{g}$ in $50 \mu \mathrm{l}$ PBS). Following cell culture, levels of Arg1 and iNOS activity in culture supernatants were determined from the production of urea and nitric oxide, respectively. Data are presented as the mean \pm standard deviation, $\mathrm{n}=6$. ${ }^{* *} \mathrm{P}<0.01$ vs. control. Arg1, arginase 1; iNOS, inducible nitric oxide synthase; OVA, ovalbumin; IM, interstitial macrophage; PBS, phosphate-buffered saline.

The existence of regulatory macrophages has previously been indicated (6). A key feature of regulatory macrophages is high-level production of the anti-inflammatory cytokine IL-10 (13). In addition, regulatory macrophages have been demonstrated to reduce inflammation and pathological changes in a mouse model of lethal endotoxemia (14) and a mouse model of EAE (15). Therefore, in contrast to the M1 and M2 macrophage phenotypes, regulatory macrophages may be crucial in modulating excessive immune responses and preventing inflammatory disease. Interestingly, it has been observed that lung macrophages from asthmatic subjects produced less IL-10 than those from healthy individuals (23). Furthermore, in severe asthma, lung macrophages have been found to produce higher levels of IL-6, IL-8 and monocyte-derived chemokines, while IL-10 was undetectable in the cells, relative to those from patients with moderate asthma (24). This suggests that in cases of severe asthma, the inhibitory functions of lung macrophages are absent, while their pro-inflammatory functions are enhanced (24). Results also indicate that under normal physiological conditions, lung IMs with regulatory characteristics serve key roles in the maintenance of respiratory tract immune homeostasis and prevention of airway allergic responses (25). In the present study, significant differences in the levels of IM IL-10 expression between asthmatic and control mice indicates that IL-10 is a potential homeostatic regulator under normal conditions. Therefore, lung IMs may lose their protective regulatory effects in OVA-induced asthmatic mice.

Macrophages may differentiate into an M2 phenotype following exposure to IL-4, IL-13 or a combination of both $(6,7)$. IL-4 and IL-13 are abundant in the lungs of asthmatic individuals, which may explain why pulmonary IMs were polarized towards the M2 state in OVA-induced asthmatic mice in the present study. However, the potential roles of M2-polarized lung IMs in the pathogenesis of asthma are currently unknown. In a previous murine model of asthma, it 
was observed that alternatively activated macrophages were involved in disease development $(19,36)$. Previous results also indicate that asthmatic mice have higher counts of pulmonary M2 macrophages relative to controls. In addition, it has been observed that increasing the number of M2 macrophages in the lungs by intratracheal instillation, prior to the induction of allergic inflammation, leads to greater airway inflammation. In patients with severe asthma, increased counts of alternatively activated macrophages have been detected in the BALF when compared with healthy controls. It has also been observed that the number of pulmonary M2 macrophages correlated with peak expiratory flow variation (20). These data suggest that alternatively activated macrophages may serve a key regulatory role in asthma. Similarly, previous results have demonstrated that adoptive transfer of IL-4 receptor (R)- $\alpha^{+/+}$macrophages, but not IL-4R $\alpha^{-/}$macrophages, by intraperitoneal injection was sufficient to strengthen Th2-dependent, OVA-induced allergic airway inflammation in mice (37). Although these results have been questioned, because it has previously been reported that IL-4R $\alpha$-dependent alternatively activated macrophages do not serve an important role in the pathology of disease by using LysM ${ }^{\text {cre }} \mathrm{IL}-4 \mathrm{R} \alpha^{-/ l o x}$ mice in ovalbumin- and house dust mite-induced allergic airway diseases (38), collectively the aforementioned findings suggest that alternatively activated macrophages are key contributors of Th2-driven airway inflammation, rather than simply bystander cells that respond to Th2 cytokines. Although all macrophages may respond to IL-4 and IL-13, it is unknown whether AMs and/or IMs transform into an alternatively activated macrophage phenotype within asthmatic individuals. In the current study, lung IMs were polarized towards an alternative activation phenotype in murine OVA-induced asthma. Therefore, M2-polarized lung IMs may contribute to a Th2 response, though this warrants further investigation.

In conclusion, the present data indicate that lung IMs undergo a phenotypic switch from a regulatory macrophage phenotype under normal conditions to an alternative activation state in OVA-induced asthmatic mice. Further studies are now warranted to identify which factors trigger the phenotypic switch of lung IMs in asthmatic individuals, and to determine if targeted therapies can be developed that modify the characteristics of lung IMs, particularly at the levels of cytokine, metabolite and enzyme expression.

\section{Acknowledgements}

The current study was supported by staff at the Center for Medical Research at the Hubei Key Laboratory of Allergy and Immune-Related Diseases (Wuhan University, Wuhan, China) and the National Natural Science Foundation of China (grant nos. 81500021 and 81270076).

\section{References}

1. Barnes PJ: Immunology of asthma and chronic obstructive pulmonary disease. Nat Rev Immunol 8: 183-192, 2008.

2. Anderson GP: Endotyping asthma: New insights into key pathogenic mechanisms in a complex, heterogeneous disease. Lancet 372: 1107-1119, 2008

3. Martinez FO, Helming L and Gordon S: Alternative activation of macrophages: An immunologic functional perspective. Annu Rev Immunol 27: 451-483, 2009.
4. Strauss-Ayali D, Conrad SM and Mosser DM: Monocyte subpopulations and their differentiation patterns during infection. J Leukoc Biol 82: 244-252, 2007.

5. Auffray C, Fogg D, Garfa M, Elain G, Join-Lambert O, Kayal S, Sarnacki S, Cumano A, Lauvau G and Geissmann F: Monitoring of blood vessels and tissues by a population of monocytes with patrolling behavior. Science 317: 666-670, 2007.

6. Gordon S and Taylor PR: Monocyte and macrophage heterogeneity. Nat Rev Immunol 5: 953-964, 2005.

7. Martinez FO, Sica A, Mantovani A and Locati M: Macrophage activation and polarization. Front Biosci 13: 453-461, 2008.

8. Mosser DM and Edwards JP: Exploring the full spectrum of macrophage activation. Nat Rev Immunol 8: 958-969, 2008.

9. Gorden S and Martinez FO: Alternative activation of macrophages: Mechanism and functions. Immunity 32: 593-604, 2010

10. Loke P, Nair MG, Parkinson J, Guiliano D, Blaxter M and Allen JE: IL-4 dependent alternative activated macrophages have a distinct in vivo gene expression phenotype. BMC Immunol 3: 7, 2002.

11. Nair MG, Cochrane DW and Allen JE: Macrophages in chronic type 2 inflammation have a novel phenotype characterized by the abundant expression of Ym1 and Fizzl that can be partly replicated in vivo. Immunol Lett 85: 173-180, 2003.

12. Sandler NG, Mentink-Kane MM, Cheever AW and Wynn TA: Global gene expression profiles during acute pathogen-induced pulmonary inflammation reveal divergent roles for Th1 and Th2 responses in tissue repair. J Immunol 171: 3655-3667, 2003.

13. Sutterwala FS, Noel GJ, Salgame P and Mosser DM: Reversal of proinflammatory responses by ligating the macrophage Fcgamma receptor type I. J Exp Med 188: 217-222, 1998.

14. Gerber JS and Mosser DM: Reversing lipopolysaccharide toxicity by ligating the macrophage $\mathrm{Fc}$ gamma receptors. J Immunol 166: 6861-6868, 2001

15. Fleming BD and Mosser DM: Regulatory macrophages: Setting the threshold for therapy. Eur J Immunol 41: 2498-2502, 2011.

16. Schneberger D, Aharonson-Raz K and Singh B: Monocyte and macrophage heterogeneity and Toll-like receptors in the lung. Cell Tissue Res 343: 97-106, 2011.

17. Holt PG, Strickland DH, Wikström ME and Jahnsen FL: Regulation of immunological homeostasis in the respiratory tract. Nat Rev Immunol 8: 142-152, 2008.

18. Martinez FO, Helming L, Milde R, Varin A, Melgert BN Draijer C, Thomas B, Fabbri M, Crawshaw A, Ho LP, et al: Genetic programs expressed in resting and IL-4 alternatively activated mouse and human macrophages: Similarities and differences. Blood 121: e57-e69, 2013.

19. Melgert BN, ten Hacken NH, Rutgers B, Timens W, Postma DS and Hylkema MN: More alternative activation of macrophages in lungs of asthmatic patients. J Allergy Clin Immunol 127: 831-833, 2011.

20. Kim EY, Battaile JT, Patel AC, You Y, Agapov E, Grayson MH, Benoit LA, Byers DE, Alevy Y, Tucker J, et al: Persistent activation of an innate immune response translates respiratory viral infection into chronic lung disease. Nat Med 14: 633-640, 2008.

21. Chupp GL, Lee CG, Jarjour N, Shim YM, Holm CT, He S, Dziura JD, Reed J, Coyle AJ, Kiener P, et al: A chitinase-like protein in the lung and circulation of patients with severe asthma. N Engl J Med 357: 2016-2027, 2007.

22. Draijer C, Robbe P, Boorsma CE, Hylkema MN and Melgert BN: Characterization of macrophage phenotypes in three murine models of house-dust-mite-induced asthma. Mediators Inflamm 2013: 632049, 2013.

23. Maneechotesuwan K, Supawita S, Kasetsinsombat K, Wongkajornsilp A and Barnes PJ: Sputum indoleamine-2, 3-dioxygenase activity is increased in asthmatic airways by using inhaled corticosteroids. J Allergy Clin Immunol 121: 43-50, 2008.

24. Fitzpatrick AM, Higgins M, Holguin F, Brown LA and Teague WG; National Institutes of Health/National Heart, Lung, and Blood Institute's Severe Asthma Research Program: The molecular phenotype of severe asthma in children. J Allergy Clin Immunol 125: 851-857.e18, 2010.

25. Bedoret D, Wallemacq H, Marichal T, Desmet C, Quesada Calvo F, Henry E, Closset R, Dewals B, Thielen C, Gustin P, et al: Lung interstitial macrophages alter dendritic cell functions to prevent airway allergy in mice. J Clin Invest 119: 3723-3738, 2009.

26. Lagranderie M, Nahori MA, Balazuc AM, Kiefer-Biasizzo H, Lapa e Silva JR, Milon G, Marchal G and Vargaftig BB: Dendritic cells recruited to the lung shortly after intranasal delivery of Mycobacterium bovis BCG drive the primary immune response towards a type 1 cytokine production. Immunology 108: 352-364, 2003. 
27. Larionov A, Krause A and Miller W: A standard curve based method for relative real time PCR data processing. BMC Bioinformatics 6: 62, 2005 .

28. Mantovani A, Sica A, Sozzani S, Allavena P, Vecchi A and Locati M: The chemokine system in diverse forms of macrophage activation and polarization. Trends Immunol 25: 677-686, 2004.

29. Gordon S: Alternative activation of macrophages. Nat Rev Immunol 3: 23-35, 2003.

30. Zhu Z, Zheng T, Homer RJ, Kim YK, Chen NY, Cohn L, Hamid Q and Elias JA: Acidic mammalian chitinase in asthmatic Th2 inflammation and IL-13 pathway activation. Science 304: 1678-1682, 2004.

31. Porcheray F, Viaud S, Rimaniol AC, Léone C, Samah B, Dereuddre-Bosquet N, Dormont D and Gras G: Macrophage activation switching: An asset for the resolution of inflammation. Clin Exp Immunol 142: 481-489, 2005.

32. Laskin DL, Sunil VR, Gardner CR and Laskin JD: Macrophages and tissue injury: Agents of defense or destruction? Annu Rev Pharmacol Toxicol 51: 267-288, 2011.

33. Stout RD and Suttles J: Functional plasticity of macrophages: Reversible adaptation to changing microenvironments. J Leukoc Biol 76: 509-513, 2004.
34. Mantovani A, Sozzani S, Locati M, Allavena P and Sica A: Macrophage polarization: Tumor-associated macrophages as a paradigm for polarized M2 mononuclear phagocytes. Trends Immunol 23: 549-555, 2002.

35. Bronte $\mathrm{V}$ and Zanovello P: Regulation of immune responses by L-arginine metabolism. Nat Rev Immunol 5: 641-654, 2005.

36. Melgert BN, Oriss TB, Qi Z, Dixon-McCarthy B, Geerlings M, Hylkema MN and Ray A: Macrophages: Regulators of sex differences in asthma? Am J Respir Cell Mol Biol 42: 595-603, 2010.

37. Ford AQ, Dasgupta P, Mikhailenko I, Smith EM, Noben-Trauth N and Keegan AD: Adoptive transfer of IL-4R $\alpha+$ macrophages is sufficient to enhance eosinophilic inflammation in a mouse model of allergic lung inflammation. BMC Immunol 13: 6, 2012.

38. Nieuwenhuizen NE, Kirstein F, Jayakumar J, Emedi B, Hurdayal R, Horsnell WG, Lopata AL and Brombacher F: Allergic airway disease is unaffected by the absence of IL-4R $\alpha$-dependent alternatively activated macrophages. J Allergy Clin Immunol 130: 743-750.e8, 2012. 